\title{
Pulmonary static inflation with $50 \%$ Xenon during cardiopulmonay bypass attenuated early postoperative inflammation in Chinese adults undergoing Stanford Type-A Acute Aortic Dissection Surgery: A pilot study
}

\author{
Mu Jin, Jiakai Lu, Weiping Cheng* \\ (*Corresponding author:ch_eng9735@sina.com) \\ Center for Anesthesiology, Beijing Anzhen Hospital, Capital Medical University, \\ Beijing, China
}

\section{INTRODUCTION 0}

Background Aortic dissection is the end stage of different pathological processes stressing on the aortic wall, which inflammation plays an important role in aortic dissection. Xenon is one of noble gases and has been recognized as an anesthetic which can exert neuroprotective and cardioprotective effects in different models by pre-, realtimeand post-conditioning.

Objective To determine the effect of a pulmonary static inflation with $50 \%$ Xenon on perioperative inflammatory cytokines in patients with acute Stanford type-A dissection surgery.

\section{METHODS \\ Design, \\ Setting, \\ and} Participants A prospective signalcenter clinical trial, recruited 100 adult patients undergoing Stanford type-A AAD surgery at an academic hospital in China(ChiCTR-ICR-5006435).All patients were divided randomly into the two groups:control group and Xenon group. In Xenon group,After Aortic crossclamping,mechanical ventilation was discontinued and pulmonary static inflation with $50 \%$ Xenon was processed to maintain air pressure $5 \mathrm{cmH} 2 \mathrm{O}$.Before 10-15 min of aortic declamping and heart resuscitation, pulmonary static inflation with $50 \%$ Xenon was stopped and then mechanical ventilation was continued.

Main outcome measures The serum variables including inflammatory and endothelial cell function were assayed.

\section{RESULTS}

There was significant effect of time and treatment-time interaction for IL- $6(p=0.000$ and $p=0.000$, respectively), IL- $10(p=0.000$ and $p=0.001$, respectively), TNF $\alpha(p=0.012$ and $p=0.025$, respectively)and $\operatorname{TXB}_{2}(p=0.000$ and $\quad p=0.001$, respectively).In first fraction(from beginning to postoperative 10 min), the value of IL-6, TNF $\alpha$ and TXB decreased $23.5 \%, 9.1 \%$ and $30.2 \%$ respectively in Xenon group, but the concurrent value increased $10.8 \%, 26.2 \%$ and $26.4 \%$ respectively in control group. In first fraction, the value of IL-10 increased $28 \%$ in Xenon group and decreased $7.5 \%$ in control group respectively. In the second (from postoperative $10 \mathrm{~min}$ to postoperative $6 \mathrm{~h}$ ) and third fractions (postoperative 6-24h), the values of IL-6,IL-10,TNF $\alpha$ and $\mathrm{TXB}_{2}$ changed quite similar in both groups. Perioperative serum level of $\mathrm{PGI}_{2}$ was relatively stable, with no significant differences between the groups.
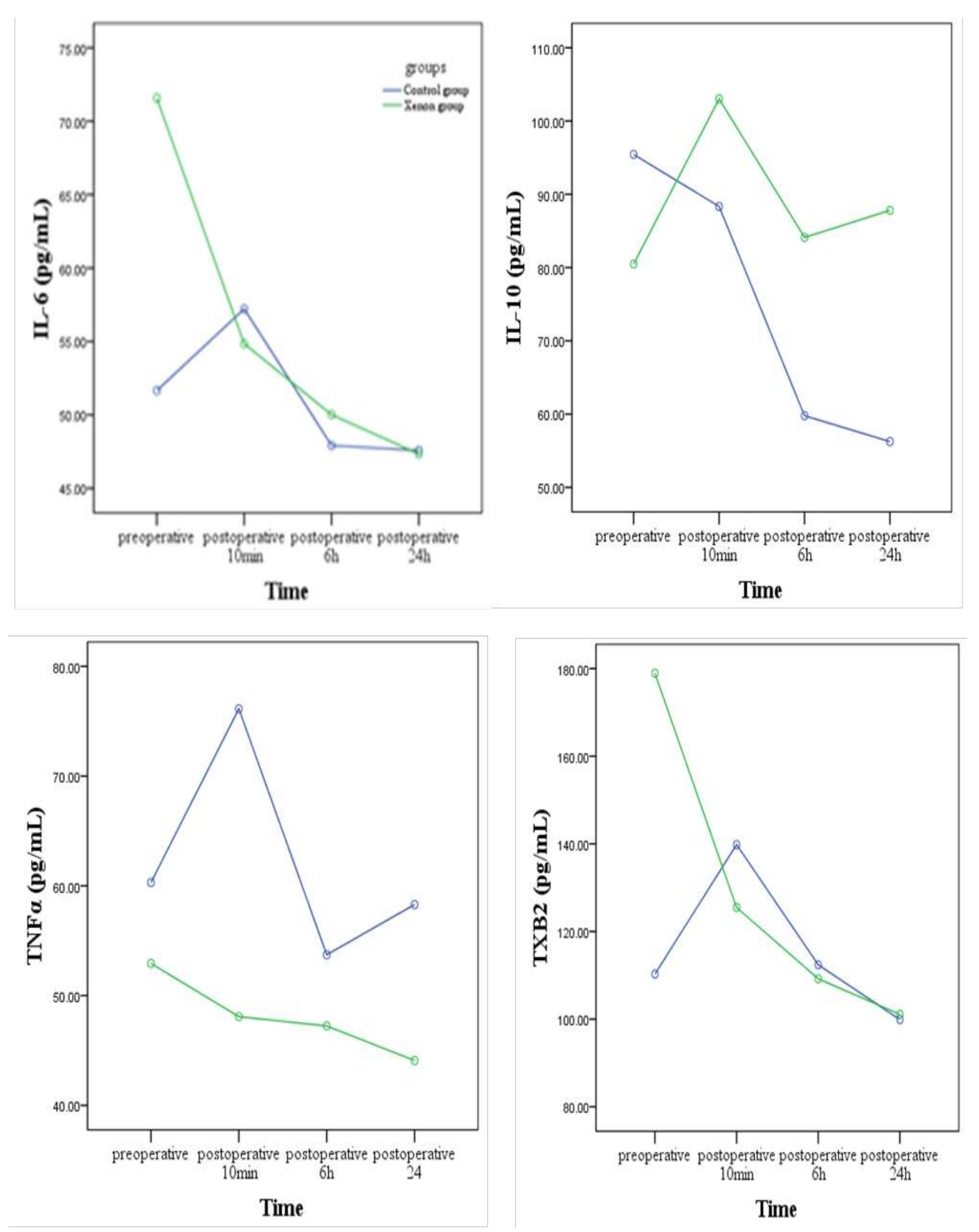
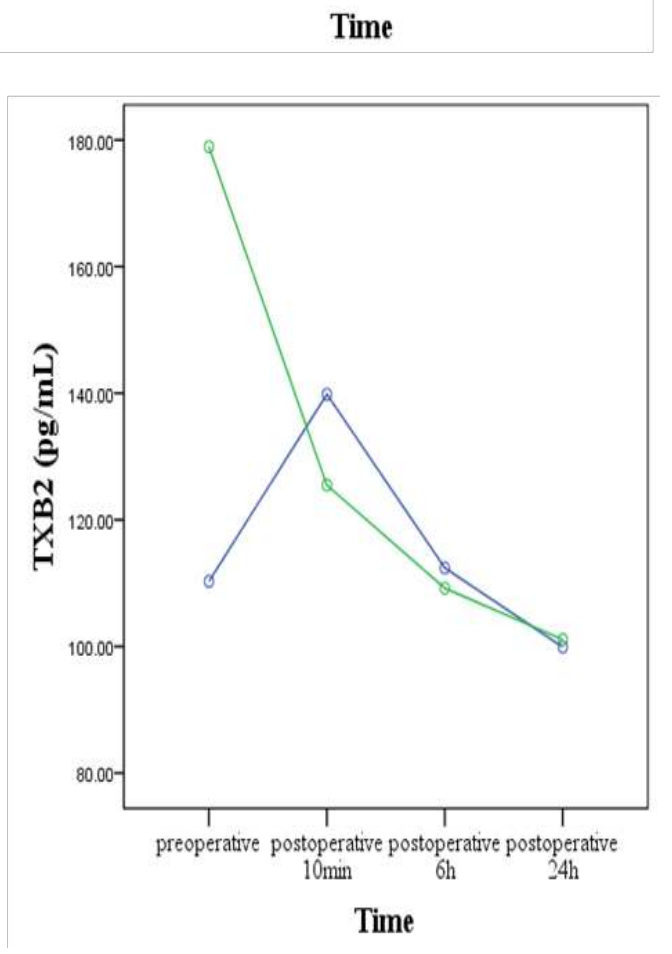

Pulmonary static inflation with $50 \%$ Xenon during Cardiopulmonay bypass could attenuate early postoperative inflammation in Chinese adults undergoing Stanford Type-A Acute Aortic Dissection Surgery.

\section{CONCLUSIONS}

\section{Flow chart}

100 adult patients undergoing Stanford type-A AAD surgery were recruited

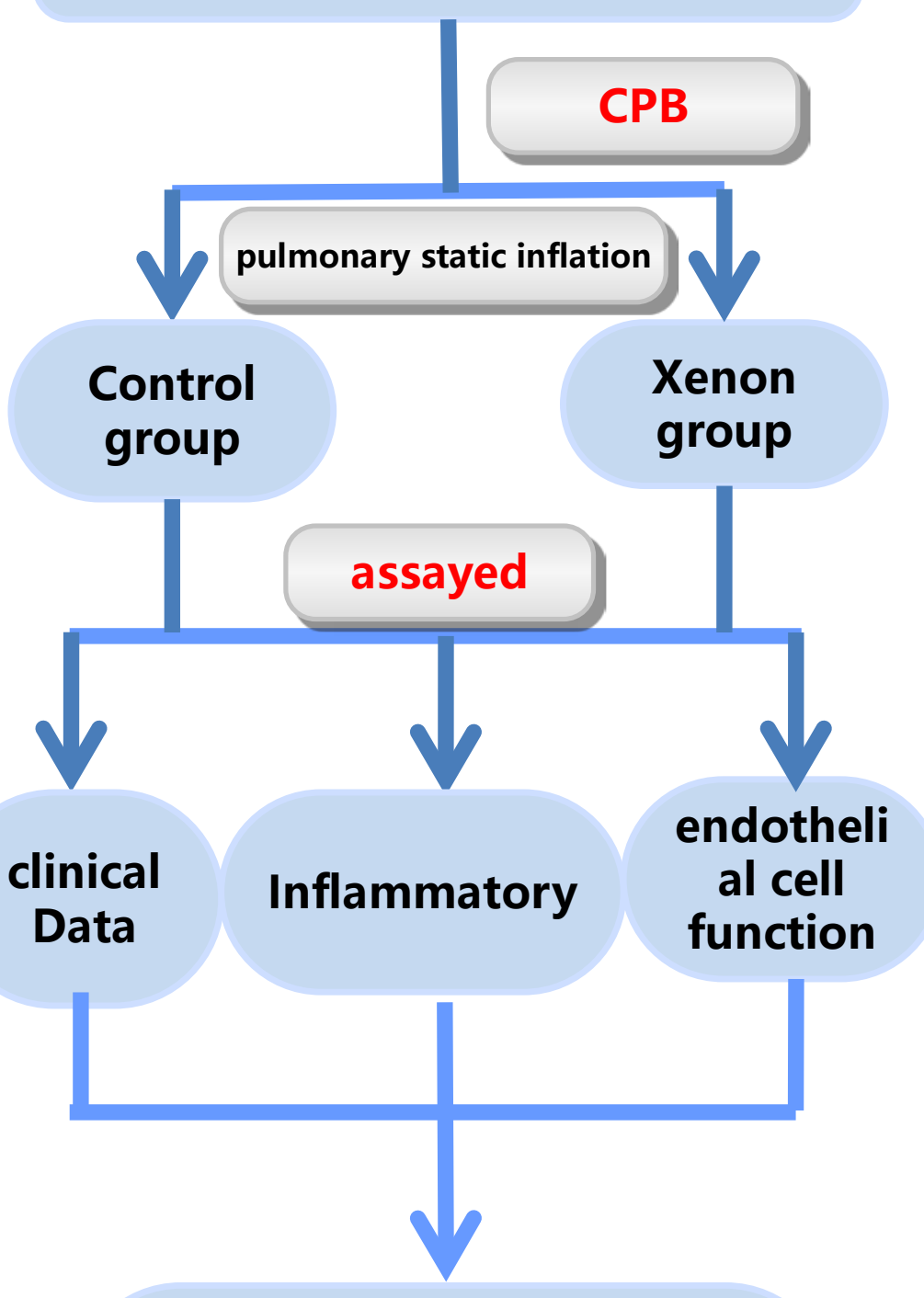

Follow up 